United Nations Educational Scientific and Cultural Organization and

International Atomic Energy Agency

THE ABDUS SALAM INTERNATIONAL CENTRE FOR THEORETICAL PHYSICS

\title{
ON WEAKLY HYPERBOLIC SPACES AND A CONVERGENCE-EXTENSION THEOREM IN WEAKLY HYPERBOLIC SPACES
}

\author{
Pham Viet Duc ${ }^{1}$ \\ Department of Mathematics, Thainguyen University, Thainguyen, Vietnam \\ and \\ The Abdus Salam International Centre for Theoretical Physics, Trieste, Italy.
}

\begin{abstract}
In this article we prove some properties of the weakly hyperbolic spaces. Moreover, a convergence-extension theorem for analytic hypersurfaces (not necessarily with normal crossings) in weakly hyperbolic spaces is given.
\end{abstract}

MIRAMARE - TRIESTE

November 2002

\footnotetext{
${ }^{1}$ phamvietduc2002@yahoo.com
} 


\section{$\S$ 1. INTRODUCTION}

In [1] O. Alehyane and H. Amal presented the concept "the weakly hyperbolic space". The first aim of this paper is to prove some properties of the weakly hyperbolic spaces. Exactly, we study the weak hyperbolicity in holomorphic fiber bundles and the normalization of a complex space by the following results:

Theorem 1. Let $(Y, \pi, X)$ be a holomorphic fiber bundle with fiber $F$. If $X$ is weakly hyperbolic and $F$ is hyperbolic then $Y$ is weakly hyperbolic.

Theorem 2. Let $(Y, \pi, X)$ be a holomorphic fiber bundle and $f: X^{\prime} \rightarrow X$ a holomorphic mapping. If $(Y, \pi, X)$ is weakly hyperbolic, then its pull-back bundle $\left(f^{-1} Y, \pi^{\prime}, X^{\prime}\right)$ is also weakly hyperbolic.

Theorem 3. Let $(Y, \pi)$ be a normalization of a complex space $X$. If $X$ is weakly hyperbolic then $Y$ is also weakly hyperbolic.

At the end of Section 2 we show the Eastwood's Lemma for the weak hyperbolicity of complex spaces and the openness of the weak hyperbolicity.

Theorem 4. Let $f: Y \rightarrow X$ be a holomorphic mapping of complex spaces. Assume that $X$ is weakly hyperbolic and that to every point $a \in X$ there is a neighbourhood $U$ of a such that $\pi^{-1}(U)$ is hyperbolic. Then $Y$ is weakly hyperbolic.

Proposition 5. Let $f: Y \rightarrow X$ be a proper holomorphic mapping of complex spaces.

i) If $X$ is weakly hyperbolic and each fiber $f^{-1}(x)$ is weakly hyperbolic for all $x \in X$, then $Y$ is weakly hyperbolic.

ii) If there is some $x_{0} \in X$ such that $f^{-1}\left(x_{0}\right)$ is weakly hyperbolic, then there exists an open neighbourhood $U$ of $x_{0}$ in $X$ such that $f^{-1}(x)$ is weakly hyperbolic for all $x \in U$. (In fact, $f^{-1}(x)$ is hyperbolic for all $x \in U$.)

Similarly as the hyperbolicity of a complex space, the weak hyperbolicity also has applications in the problem of extending holomorphic mappings [1]. In the next section, we prove a convergence-extension theorem in weakly hyperbolic complex spaces. Since this is a new concept, we hope that one can find more applications.

The convergence-extension theorem of Noguchi (see [4] or [7]) is stated as follows

Theorem. Let $X$ be relatively compact and hyperbolically imbedded into a complex space $Y$. Let $M$ be a complex manifold and $A$ an analytic hypersurface of $M$ with only normal crossings. Suppose that $\left\{f_{j}: M \backslash A \rightarrow X\right\}_{j=1}^{\infty}$ is a sequence of holomorphic mappings which converges uniformly on compact subsets of $M \backslash A$ to a holomorphic mapping $f: M \backslash A \rightarrow X$.

Then there are unique holomorphic extensions $\bar{f}_{j}: M \rightarrow Y$ of $f_{j}$ and $\bar{f}: M \rightarrow Y$ of $f$ 
over $M$. Moreover, $\left\{\bar{f}_{j}\right\}_{j=1}^{\infty}$ converges uniformly on compact subsets of $M$ to $\bar{f}$.

Recently, several Noguchi-type convergence-extension theorems for analytic hypersurfaces of complex manifolds have been obtained by various authors (see [5], [7], [13]). In particular, in [13] D.D. Thai and P. N. Mai have proved the above theorem in case that $X$ is a complex subspace of a hyperbolic complex space $Y$ such that $X$ has $\Delta^{*}$-EP for $Y$ and $A$ is an arbitrary analytic hypersurface of a complex manifold $M$.

The next aim of the article is to replace the hyperbolicity of the complex space $Y$ in the above result by the weak hyperbolicity of $X$ for $Y$. More precisely, we prove the following

Theorem 5. Let $X$ be a complex subspace of a complex space $Y$. Suppose that $X$ is weakly hyperbolic for $Y$ and has $\Delta^{*}-E P$ for $Y$. Let $A$ be any analytic hypersurface of a complex manifold $M$. Let $\left\{f_{j}: M \backslash A \rightarrow X\right\}_{j=1}^{\infty}$ be a sequence of holomorphic mappings which converges uniformly on compact subsets of $M \backslash A$ to a holomorphic mapping $f: M \backslash A \rightarrow X$.

Then there are unique holomorphic extensions $\bar{f}_{j}: M \rightarrow Y$ and $\bar{f}: M \rightarrow Y$ of $f_{j}$ and $f$ respectively over $M$. Moreover, $\left\{\bar{f}_{j}\right\}_{j=1}^{\infty}$ converges uniformly on compact subsets of $M$ to $\bar{f}$.

Finally, we generalise, partly, a theorem of Noguchi by the following

Corollary 2. Let $X$ be relatively compact and hyperbolically imbedded into a complex space $Y$. Let $M$ be a complex manifold and $A$ an analytic hypersurface of $M$. Then every holomorphic mapping $f: M \backslash A \rightarrow X$ extends to a holomorphic mapping $\bar{f}: M \rightarrow Y$.

\section{$\S$ 2. SOME PROPERTIES OF THE WEAKLY HYPERBOLIC SPACES}

For every positive number $r$, put $\Delta_{r}=\{z \in \mathbb{C}:|z|<r\}, \Delta_{1}=\Delta$.

Let $X$ be a complex space and the Kobayashi pseudodistance on $X$ is denoted by $d_{X}$.

Definition 1. A complex space $X$ is said to be hyperbolic (in the sense of Kobayashi) if $d_{X}$ is a distance on $X$, i.e., $d_{X}(x, y)>0$ if $x \neq y$.

A complex subspace $X$ is said to be hyperbolically imbedded in a complex space $Y$ if for $x, y \in \bar{X}$ and $x \neq y$, there exist open neighbourhoods $U$ of $x$ and $V$ of $y$ in $Y$ such that

$$
d_{X}(U \cap X, V \cap X)>0 .
$$

If $X=\Delta$, the Kobayashi distance coincides with Poincaré metric on $\Delta$,

$$
d_{\Delta}(z, w)=\rho_{\Delta}(z, w) .
$$

If $X$ is hyperbolic then $d_{X}$ induces the natural topology on $X$.

For details concerning hyperbolic complex spaces we refer the readers to the books [3], [4], [5] and [7]. 
Definition 2. Let $X$ be a complex space. $X$ is called to be weakly hyperbolic if for every compact subset $K \subset X$, there exists an open neighbourhood $U$ of $K$ in $X$ which satisfies the following two properties:

i) $U$ is hyperbolic;

ii) for all $f \in \operatorname{Hol}(\Delta, X) \cap \mathcal{C}(\bar{\Delta}, X)$, if $f(\partial \Delta) \subset K$ then $f(\bar{\Delta}) \subset U$.

Here, we denote $\operatorname{Hol}(\Delta, X)$ the space of holomorphic mappings from $\Delta$ into $X$ which is equipped with the compact-open topology.

Examples. (see [1])

1. Every hyperbolic space is weakly hyperbolic.

2. If $X$ is a complex space with a strictly plurisubharmonic function, then $X$ is weakly hyperbolic.

3. If $X$ is a compact complex space, then $X$ is weakly hyperbolic if and only if $X$ is hyperbolic.

4. If $\widetilde{X}$ is a holomorphic covering of an analytic space $X$, then if $X$ is weakly hyperbolic so is $\widetilde{X}$.

5. Let $\left\{z_{k}\right\} \subset \Delta,\left\{z_{k}\right\} \rightarrow 0$, and $\left\{\alpha_{k}\right\},\left\{\delta_{k}\right\} \subset(0,1)$ such that

(i) $\sum_{k \geq 1} \alpha_{k} \log \frac{1}{z_{k} \mid}<\infty$, and

(ii) $\lim _{k \rightarrow \infty} \alpha_{k} \log \frac{1}{\delta_{k}}=+\infty$.

In $\mathbb{C}^{2}$ consider the domain $X:=\left\{(z, w) \in \mathbb{C}^{2}:|z|<1,|w|<e^{-u(z)}\right\}$, where $u(z):=$ $\sum_{k} \alpha_{k} \log \left(\delta_{k}^{2}+\left|z-z_{k}\right|^{2}\right)$. We have that $X$ is weakly hyperbolic but not hyperbolic (see [1] and [14]).

6. $\mathbb{C}^{n}$ is weakly hyperbolic.

Indeed, take $K$ a compact subset of $\mathbb{C}^{n}$. There is $R>0$ such that $K \subset \Delta_{R}^{n} \subset \mathbb{C}^{n}$. We have that $\Delta_{R}^{n}$ is hyperbolic. Suppose that $f \in \operatorname{Hol}\left(\Delta, \mathbb{C}^{n}\right) \cap C\left(\bar{\Delta}, \mathbb{C}^{n}\right)$ such that $f(\partial \Delta) \subset K \subset \Delta_{R}^{n}$. We can write $f=\left(f_{1}, \ldots, f_{n}\right)$, where $f_{i} \in \operatorname{Hol}(\Delta, \mathbb{C}) \cap C(\bar{\Delta}, \mathbb{C})$. Then we deduce $\left|f_{i}(\partial \Delta)\right| \leq$ $R, \forall i=1, \ldots, n$. By the maximum principle it follows that $\left|f_{i}(\bar{\Delta})\right| \leq R, \forall i=1, \ldots, n$. Therefore $f(\bar{\Delta}) \subset \Delta_{R}^{n}$. Q.E.D

Now we prove some properties of weakly hyperbolic spaces which are useful for the next results.

Proposition 1. Let $X$ be a closed or open complex subspace of a complex space $Y$. If $Y$ is weakly hyperbolic then $X$ is also weakly hyperbolic.

Proof. Let $K$ be a compact subset of $X$. Then $K$ is compact in $Y$. Since $Y$ is weakly 
hyperbolic, there exists an open hyperbolic neighbourhood $U$ of $K$ in $Y$ such that $U$ satisfies the condition ii) in Definition 2. Thus we get $U \cap X$ is an open hyperbolic neighbourhood of $K$ in $X$. We have to prove that $U \cap X$ satisfies ii) in Definition 2. Let $f \in \operatorname{Hol}(\Delta, X) \cap \mathcal{C}(\bar{\Delta}, X)$ and $f(\partial \Delta) \subset K$. Consider $f \in \operatorname{Hol}(\Delta, Y) \cap \mathcal{C}(\bar{\Delta}, Y)$, we have $f(\bar{\Delta}) \subset U$, so $f(\bar{\Delta}) \subset U \cap X$. Hence $X$ is weakly hyperbolic. Q.E.D

Remark. Every $n$ - dimensional Stein manifold is weakly hyperbolic.

Indeed, by the Embedding Theorem in [2] $X$ can be biholomorphically mapped onto a closed complex submanifold of $\mathbb{C}^{2 n+1}$. So Proposition 1 and Example 6 imply that $X$ is weakly hyperbolic. Q.E.D

Proposition 2. If $X_{1}, X_{2}$ are weakly hyperbolic then $X_{1} \times X_{2}$ is also weakly hyperbolic.

Proof. Given $K$ a compact subset of $X_{1} \times X_{2}$. Then $K_{i}:=\operatorname{pr}_{i}(K)$ are compact subsets of $X_{i}(\mathrm{i}=1,2)$ respectively, where $\mathrm{pr}_{i}: X_{1} \times X_{2} \rightarrow X_{i}$ are projections. Hence, there exist open hyperbolic neighbourhoods $U_{i}$ of $K_{i}$ in $X_{i}$ respectively such that the condition ii) in Definition 2 is satisfied $\left(^{*}\right)$. Clearly $U_{1} \times U_{2}$ is an open hyperbolic neighbourhood of $K_{1} \times K_{2}$ in $X_{1} \times X_{2}$. Let $f \in \operatorname{Hol}\left(\Delta, X_{1} \times X_{2}\right) \cap \mathcal{C}\left(\bar{\Delta}, X_{1} \times X_{2}\right)$ and $f(\partial \Delta) \subset K$. Then $f_{i}(\partial \Delta) \subset K_{i}$, where $f_{i}(\partial \Delta):=$ $\operatorname{pr}_{i}(f(\partial \Delta)), i=1,2$. By $\left(^{*}\right)$ we have $f_{i}(\bar{\Delta}) \subset U_{i}, i=1,2$. Thus $f(\bar{\Delta}) \subset f_{1}(\bar{\Delta}) \times f_{2}(\bar{\Delta}) \subset U_{1} \times U_{2}$. This implies that $X_{1} \times X_{2}$ is weakly hyperbolic. Q.E.D

Now we study the weak hyperbolicity of holomorphic fiber bundles.

Theorem 1. Let $(Y, \pi, X)$ be a holomorphic fiber bundle with fiber $F$. If $X$ is weakly hyperbolic and $F$ is hyperbolic then $Y$ is weakly hyperbolic.

Proof. Let $K$ be a compact subset of the complex space $Y$. Since $\pi: Y \rightarrow X$ is holomorphic, then $\pi(K) \subset X$ is compact. By the weak hyperbolicity of $X$, there exists an open neighbourhood $U$ of $\pi(K)$ in $X$ which satisfies conditions i) and ii) in Definition $2\left(^{*}\right)$.

Since $\pi(K) \subset U$, then $K \subset \pi^{-1}(U)$ and we have $\pi^{-1}(U)$ is hyperbolic: indeed, for each point $x \in U$ there exists an open neighbourhood $V(x) \subset U$ such that $\pi^{-1}(V)=V \times F$. Since $U$ is hyperbolic we have that $V$ is hyperbolic. By hypothesis $F$ is hyperbolic, it implies that $\pi^{-1}(V)$ is hyperbolic. By Eastwood's Lemma [5] we get that $\pi^{-1}(U)$ is hyperbolic.

In order to prove condition ii) of Definition 2, let $f \in \operatorname{Hol}(\Delta, Y) \cap \mathcal{C}(\bar{\Delta}, Y)$ and suppose $f(\partial \Delta) \subset K$. We have to prove that

$$
f(\bar{\Delta}) \subset \pi^{-1}(U)
$$

Since $f(\partial \Delta) \subset K$, then $\pi \circ f(\partial \Delta) \subset \pi(K)$. By $\left(^{*}\right)$ we deduce

$$
\pi \circ f(\bar{\Delta}) \subset U .
$$

Hence

$$
f(\bar{\Delta}) \subset \pi^{-1}\left(\pi \circ f(\bar{\Delta}) \subset \pi^{-1}(U) .\right.
$$


Therefore $Y$ is weakly hyperbolic. Q.E.D

Remark. In the case where $(Y, \pi, X)$ is trivial we have that $X$ and $F$ are weakly hyperbolic if and only if $Y$ is weakly hyperbolic.

This follows from Propositions 1 and 2. Q.E.D

Definition 3. A holomorphic fiber bundle $(Y, \pi, X)$ is said to be weakly hyperbolic at $x \in X$ if $x$ has a neighbourhood $U$ in $X$ such that $Y_{U}=\pi^{-1}(U)$ is weakly hyperbolic, and it is weakly hyperbolic if it is weakly hyperbolic at every point $x \in X$.

Theorem 2. Let $(Y, \pi, X)$ be a holomorphic fiber bundle and $f: X^{\prime} \rightarrow X$ a holomorphic mapping. If $(Y, \pi, X)$ is weakly hyperbolic, then its pull-back bundle $\left(f^{-1} Y, \pi^{\prime}, X^{\prime}\right)$ is also weakly hyperbolic.

Proof. Set $Y^{\prime}=f^{-1} Y$. Given a point $x^{\prime} \in X^{\prime}$, by hypothesis, let $U$ be a neighbourhood of $f\left(x^{\prime}\right)$ in $X$ such that $Y_{U}$ is weakly hyperbolic. Let $U^{\prime}$ be a hyperbolic neighbourhood of $x^{\prime}$ in $X^{\prime}$ such that $U^{\prime} \subset f^{-1}(U)$. We shall prove that $Y_{U^{\prime}}^{\prime}=\pi^{\prime-1}\left(U^{\prime}\right)$ is weakly hyperbolic.

Let $K$ be a compact subset in $Y_{U^{\prime}}^{\prime}$, then $K \subset K_{1} \times K_{2}$ where $K_{1}, K_{2}$ are compact subsets in $U^{\prime}$ and $Y_{U}$ respectively. Since $Y_{U}$ is weakly hyperbolic, there exists a hyperbolic neighbourhood $V$ of $K_{2}$ in $Y_{U}$ such that the condition ii) of Definition 2 is satisfied $\left(^{*}\right)$. Therefore $\left(U^{\prime} \times V\right) \cap Y_{U^{\prime}}^{\prime}$ is a hyperbolic neighbourhood of $K$ in $Y_{U^{\prime}}^{\prime}$.

Suppose that $g \in \operatorname{Hol}\left(\Delta, Y_{U^{\prime}}^{\prime}\right) \cap C\left(\bar{\Delta}, Y_{U^{\prime}}^{\prime}\right)$ and $g(\partial \Delta) \subset K$. Then we can write $g(\partial \Delta)=$ $\left(g_{1}(\partial \Delta), g_{2}(\partial \Delta)\right) \subset K_{1} \times K_{2}$. Since $U^{\prime}$ is hyperbolic, and hence weakly hyperbolic, and by $\left(^{*}\right)$ we have that $\left(g_{1}(\bar{\Delta}), g_{2}(\bar{\Delta})\right) \subset\left(U^{\prime} \times V\right) \cap Y_{U^{\prime}}^{\prime}$. Hence $g(\bar{\Delta}) \subset\left(U^{\prime} \times V\right) \cap Y_{U^{\prime}}^{\prime}$.

It implies that $\left(Y^{\prime}, \pi^{\prime}, X^{\prime}\right)$ is weakly hyperbolic. Q.E.D

Proposition 3. Let $\left(V, \pi_{V}, X\right)$ and $\left(W, \pi_{W}, X\right)$ be holomorphic fiber bundles. If they are weakly hyperbolic then the Whitney sum $(V \oplus W, \pi, X)$ is also weakly hyperbolic.

Proof. For every $x \in X$, by hypothesis, there is a neighbourhood $U$ of $x$ in $X$ such that $V_{U}=\pi_{U}^{-1}(U)$ and $W_{U}=\pi_{W}^{-1}(U)$ are weakly hyperbolic. Since $(V \oplus W)_{U}$ is a complex subspace of $V_{U} \times W_{U}$, then by Propositions 1 and 2 we deduce that $(V \oplus W)_{U}$ is weakly hyperbolic. It follows that $(V \oplus W, \pi, X)$ is weakly hyperbolic. Q.E.D

Definition 4. (see [3]) A complex space $X$ is said to be normal at a point $a \in X$ if the ring $\mathcal{O}_{X, a}$ of germs of holomorphic functions at $a$ is integrally closed in its ring of quotients. If $X$ is normal at every point of $X$, then $X$ is said to be normal.

A normalization of a complex space $X$ is a pair $(Y, \pi)$ consisting of a normal complex space $Y$ and a surjective holomorphic mapping $\pi: Y \rightarrow X$ such that

i) $\pi: Y \rightarrow X$ is proper and $\pi^{-1}(a)$ is finite for every $a \in X$; 
ii) If $S$ is the set of singular points of $X$, then $Y \backslash \pi^{-1}(S)$ is dense in $Y$ and $\pi: Y \backslash \pi^{-1}(S) \rightarrow$ $X \backslash S$ is biholomorphic.

Every complex space $X$ has a unique (up to an isomorphism) normalization $(Y, \pi)$ (see $[8],[6, \mathrm{p} .118])$.

The following Theorem shows that the weak hyperbolicity is preserved by passing to the normalization of a complex space.

Theorem 3. Let $(Y, \pi)$ be a normalization of a complex space $X$. If $X$ is weakly hyperbolic then $Y$ is also weakly hyperbolic.

This Theorem is deduced immediately from the following Proposition.

Proposition 4. Let $\pi: Y \rightarrow X$ be a proper holomorphic mapping of complex spaces such that $\pi^{-1}(a)$ is finite for every $a \in X$. If $X$ is weakly hyperbolic, so is $Y$.

Proof. Let $K$ be a compact subset of the complex space $Y$. Since $\pi: Y \rightarrow X$ is holomorphic, we have that $\pi(K) \subset X$ is compact. By hypothesis $X$ is weakly hyperbolic, there exists an open neighbourhood $U$ of $\pi(K)$ in $X$ which satisfies conditions i) and ii) of Definition 2 $(*)$.

Since $\pi(K) \subset U$, then $K \subset \pi^{-1}(U)$. By Theorem $4.1[3$, p.101] and since $U$ is hyperbolic, we get that $\pi^{-1}(U)$ is hyperbolic.

Let $f \in \operatorname{Hol}(\Delta, Y) \cap \mathcal{C}(\bar{\Delta}, Y)$ and suppose $f(\partial \Delta) \subset K$. Then $\pi \circ f(\partial \Delta) \subset \pi(K)$. By $\left({ }^{*}\right)$ we deduce that $\pi \circ f(\bar{\Delta}) \subset U$. It follows that $\pi^{-1}(\pi \circ f(\bar{\Delta})) \subset \pi^{-1}(U)$. Hence

$$
f(\bar{\Delta}) \subset \pi^{-1}(\pi \circ f(\bar{\Delta})) \subset \pi^{-1}(U)
$$

Therefore $Y$ is weakly hyperbolic. Q.E.D

Theorem 4. (Eastwood's Lemma for the weak hyperbolicity)(see [5])

Let $\pi: Y \rightarrow X$ be a holomorphic mapping of complex spaces. Assume that $X$ is weakly hyperbolic and that to every point $a \in X$ there is a neighbourhood $U$ of a such that $\pi^{-1}(U)$ is hyperbolic. Then $Y$ is weakly hyperbolic.

Proof. Let $K$ be a compact subset in $Y$. Then $\pi(K)$ is compact in $X$. By the weak hyperbolicity of $X$, there exists an open hyperbolic neighbourhood $U$ of $\pi(K)$ in $X$ which satisfies condition ii) of Definition 2.

We have that $\pi^{-1}(U)$ is an open hyperbolic neighbourhood of $K$ in $Y$ : Indeed, by hypothesis and applying Eastwood's Lemma to the hyperbolic neighbourhood $U$ we deduce that $\pi^{-1}(U)$ is hyperbolic.

In the same way as in the Proof of Proposition 4 we have that condition ii) of Definition 2 is satisfied. Hence $Y$ is weakly hyperbolic. Q.E.D 
The following Proposition shows that the weak hyperbolicity is open under proper holomorphic mappings.

Proposition 5. Let $f: Y \rightarrow X$ be a proper holomorphic mapping of complex spaces.

i) If $X$ is weakly hyperbolic and each fiber $f^{-1}(x)$ is weakly hyperbolic for all $x \in X$, then $Y$ is weakly hyperbolic.

ii) If there is some $x_{0} \in X$ such that $f^{-1}\left(x_{0}\right)$ is weakly hyperbolic, then there exists an open neighbourhood $U$ of $x_{0}$ in $X$ such that $f^{-1}(x)$ is weakly hyperbolic for all $x \in U$. (In fact, $f^{-1}(x)$ is hyperbolic for all $x \in U$.)

Proof. i) Let $K$ be a compact subset of $Y$. Then $f(K)$ is compact in $X$. By hypothesis, there exists an open neighbourhood $U$ of $f(K)$ in $X$ such that $U$ satisfies conditions i) and ii) of Definition 2.

First we prove that $f^{-1}(U)$ is hyperbolic. By hypothesis and Example $4 \S 2, f^{-1}(x)$ is hyperbolic for all $x \in U$. Since $U$ is hyperbolic, applying Proposition 3.1 [5, p.72] we deduce that $f^{-1}(U)$ is hyperbolic. The condition ii) is implied by the same arguments as in Proof of Proposition 4. Thus $Y$ is weakly hyperbolic.

ii) Since $f$ is proper, $f^{-1}\left(x_{0}\right)$ is compact. Combining with the hypothesis $f^{-1}\left(x_{0}\right)$ is weakly hyperbolic we get that $f^{-1}\left(x_{0}\right)$ is hyperbolic. By Theorem $2.2([5], \mathrm{p} .68)$, there is an open hyperbolic neighbourhood $V$ of $f^{-1}\left(x_{0}\right)$. It implies that there is some open neighbourhood $U$ of $x_{0}$ in $X$ such that $f^{-1}(U) \subset V$ : Indeed, if it is not, then there exists a sequence $\left\{y_{n}\right\} \subset Y \backslash V$ $\left(^{*}\right)$ such that $f\left(y_{n}\right)=x_{n} \rightarrow x_{0}$. Let $K$ be a compact neighbourhood of $x_{0}$ in $X$. Then by the properness of $f, f^{-1}(K)$ is compact in $Y$. Since $x_{n} \rightarrow x_{0}$ we have $x_{n} \in K, \forall n \geq n_{0}$. Hence there exists a subsequence $\left\{y_{n_{k}}\right\} \subset\left\{y_{n}\right\}$ such that $y_{n_{k}} \rightarrow y_{0}$. Since $f$ is continuous, $f\left(y_{0}\right)=\lim _{k \rightarrow \infty} f\left(y_{n_{k}}\right)=\lim _{k \rightarrow \infty} x_{n_{k}}=x_{0}$. Therefore $y_{0} \in f^{-1}\left(x_{0}\right) \subset V$. We have $y_{n_{k}} \rightarrow y_{0} \in V$, then there exists $k_{0} \in \mathbb{N}$ such that $y_{n_{k}} \in V$ for all $k \geq k_{0}$, which is in contradiction to $\left({ }^{*}\right)$. Therefore, $f^{-1}(x) \subset f^{-1}(U) \subset V$ is hyperbolic for all $x \in U$, thus concluding the proof of the theorem.

Q.E.D

\section{§ 3. A CONVERGENCE-EXTENSION THEOREM IN WEAKLY HYPERBOLIC SPACES}

Definition 5. Let $X$ be a complex subspace of a complex space $Y$ and $\Delta^{*}:=\Delta \backslash\{0\}$. We say that $X$ has the $\Delta^{*}$-EP for $Y$ if every holomorphic mapping $f$ from $\Delta^{*}$ into $X$ extends to a holomorphic mapping $\bar{f}$ from $\Delta$ into $Y$. If $X$ has the $\Delta^{*}$-EP for itself then $X$ is said to have the $\Delta^{*}$-EP.

Examples. 1) By a theorem of Kobayashi [4, Thm 6.3.7, p.284], if $X$ is relatively compact and 
hyperbolically imbedded into $Y$ then $X$ has the $\Delta^{*}$-EP for $Y$. In particular, if $X$ is compact hyperbolic then $X$ has $\Delta^{*}$-EP.

2) It is easy to see from the Riemann extension theorem that if $D$ is a bounded domain in $\mathbb{C}^{n}$ and $\Omega$ is an open neighbourhood of $\bar{D}$ in $\mathbb{C}^{n}$ then $D$ has the $\Delta^{*}$-EP for $\Omega$.

For details concerning the $\Delta^{*}$-EP we refer the readers to [10], [11] and [14].

Definition 6. An open complex subspace $X$ of a complex space $Y$ is said to be weakly discconvex for $Y$ if every sequence $\left\{f_{n}\right\} \subset \operatorname{Hol}(\Delta, Y)$ converges in $\operatorname{Hol}(\Delta, Y)$ whenever the sequence $\left\{\left.f_{n}\right|_{\Delta^{*}}\right\} \subset \operatorname{Hol}\left(\Delta^{*}, X\right)$ converges in $\operatorname{Hol}\left(\Delta^{*}, X\right)$. If $X$ is weakly disc-convex for itself then $X$ is said to be weakly disc-convex.

Remarks. a) From the theorems of Montel and Kiernan (see [9]) we get the following implications:

Complete hyperbolic $\Longrightarrow$ taut $\Longrightarrow$ weakly disc-convex.

The converse assertions are not true in general (see [9]).

For details concerning the (weak) disc-convexity we refer the readers to [12].

b) If $X$ is relatively compact and hyperbolically imbedded in $Y$, then $X$ is weakly discconvex for $Y$.

Indeed, this is deduced from Noguchi's theorem on $\Delta$ (see [5, Thm. 4.1, p.56]) and Definition 6. Q.E.D

Definition 7. Let $X$ be an open complex subspace of a complex space $Y . X$ is said to be weakly hyperbolic for $Y$ if for every compact subset $K \subset \bar{X}$, there exists an open neighbourhood $U$ of $K$ in $Y$ which satisfies the following

i) $U$ is hyperbolic;

ii) for all $f \in \operatorname{Hol}(\Delta, Y) \cap \mathcal{C}(\bar{\Delta}, Y)$, if $f(\partial \Delta) \subset K$ then $f(\bar{\Delta}) \subset U$.

Remark. When $X=Y$ we get the concept of the weak hyperbolicity of the complex space $X$.

The following is a relation between the weak disc-convexity and the $\Delta^{*}$-EP in a weakly hyperbolic complex subspace $X$ for a complex space $Y$.

Proposition 6. Let $X$ be a weakly hyperbolic complex subspace for a complex space $Y$. If $X$ has the $\Delta^{*}$-EP for $Y$ then $X$ is weakly disc-convex for $Y$.

Proof. Let $\left\{f_{n}\right\}_{n \geq 1} \subset \operatorname{Hol}(\Delta, Y)$ converge in $\operatorname{Hol}\left(\Delta^{*}, X\right)$ to $f \in \operatorname{Hol}\left(\Delta^{*}, X\right)$. Since $X$ has the $\Delta^{*}$-EP for $Y$, there exists $g \in \operatorname{Hol}(\Delta, Y)$ such that $g=f$ on $\Delta^{*}$.

Put $x_{0}=g(0)$. By the continuity of $g$ we get that $x_{0} \in \bar{X}$. Put $K:=\overline{V \cap X}$, where $V$ is a relatively compact neighbourhood of $x_{0}$ in $Y$. By the hypothesis that $X$ is weakly hyperbolic for $Y$, there is an open hyperbolic neighbourhood $U \subset Y$ of $K$ which satisfies ii) in Definition 7. 
Take $s \in(0,1)$ such that $g\left(\bar{\Delta}_{s}\right) \subset V$. So we have $g\left(\partial \Delta_{s}\right) \subset g\left(\bar{\Delta}_{s}\right) \subset V$. Therefore

$$
f_{n}\left(\partial \Delta_{s}\right) \rightarrow f\left(\partial \Delta_{s}\right)=g\left(\partial \Delta_{s}\right) \subset V
$$

On the other hand, $f_{n}, f \in \operatorname{Hol}\left(\Delta^{*}, X\right)$ then $f_{n}\left(\partial \Delta_{s}\right) \rightarrow f\left(\partial \Delta_{s}\right) \subset V \cap X \subset K$. It implies that there exists $n_{0} \geq 1$ such that $f_{n}\left(\partial \Delta_{s}\right) \subset K$ for all $n \geq n_{0}$ : Indeed, since $f\left(\partial \Delta_{s}\right)$ is compact and $V \cap X$ is open in $Y$ we have $\operatorname{dist}\left(f\left(\partial \Delta_{s}\right), \partial(V \cap X)\right)=\delta>0$, where dist is a distance function with respect to some metric in $Y$. By hypothesis that $f_{n}\left(\partial \Delta_{s}\right)$ converges uniformly to $f\left(\partial \Delta_{s}\right)$, there exists $n_{0} \geq 1$ such that for all $n \geq n_{0}$ we have $\operatorname{dist}\left(f_{n}(x), f(x)\right)<\delta \quad \forall x \in \partial \Delta_{s}$. Therefore $f_{n}\left(\partial \Delta_{s}\right) \subset V \cap X$ for all $n \geq n_{0}$. Since $X$ is weakly hyperbolic for $Y$, we have

$$
f_{n}\left(\bar{\Delta}_{s}\right) \subset U \text { for all } n \geq n_{0}
$$

By abuse of notation let us denote by $f_{n}$ also the mapping $\left.f_{n}\right|_{\Delta_{s}}$, then we get $\left\{f_{n}\right\} \subset \operatorname{Hol}\left(\Delta_{s}, U\right)$. Since $U$ is hyperbolic, by Ascoli's theorem, to prove the relatively compactness of $\left\{f_{n}\right\}_{n \geq 1}$ in $\operatorname{Hol}\left(\Delta_{s}, U\right)$ it suffices to prove that the subset $X_{t}:=\left\{f_{n}(t), n \geq 1\right\}$ is relatively compact in $U$ for all $t \in \Delta_{s}$.

If $t \in \Delta_{s}^{*}$, the uniformly convergence on the compact subsets of $\Delta_{s}^{*}$ implies $X_{t} \Subset U$.

For $t=0$, suppose that $X_{0}$ is not relatively compact in $U$. Take $\left\{K_{i}\right\}_{i \in \mathbb{N}^{*}}$ an exhaustive sequence of compact subsets of $U$, i.e., $K_{i} \subset \operatorname{int}\left(K_{i+1}\right)$ for all $i$ and $U=\bigcup_{i} K_{i}$. Then there exist two increasing sequences $n_{i}, m_{i} \in \mathbb{N}^{*}$ such that $f_{n_{i}}(0) \in K_{m_{i+1}} \backslash K_{m_{i}}$ for all $i \geq 1$.

Since $g$ is continuous, by the same argument as above, there is $r \in(0,1)$ such that $g\left(\Delta_{r}\right) \subset K$. By the compactness of $K \subset U$, there is $p \in \mathbb{N}^{*}$ such that $K \subset K_{m_{p}}$. Then for all $q \geq p+2$ we have

$$
f_{n_{q}}(0) \in U \backslash K_{m_{p+1}} \text { and } \mathbb{B}\left(f_{n_{q}}(0), \varepsilon\right) \subset U \backslash K_{m_{p+1}},
$$

where $\varepsilon:=\frac{1}{2} d_{X}\left(\partial K_{m_{p+1}}, \partial K_{m_{p+2}}\right)$. Since $U$ is hyperbolic, the family $\left\{f_{n} ; n \geq n_{0}\right\}$ is equicontinuous. Hence there exists $s^{\prime} \in(0, s)$ such that

$$
d_{X}\left(f_{n}(z), f_{n}(0)\right)<\varepsilon \quad \forall z \in \Delta_{s^{\prime}}, \forall n \geq 1
$$

Put $\delta:=\min \left(\frac{s^{\prime}}{2}, \frac{r}{2}\right)$, then we have

$$
g\left(\partial \Delta_{\delta}\right)=f\left(\partial \Delta_{\delta}\right) \subset K \text { and } f_{n_{q}}\left(\partial \Delta_{\delta}\right) \subset U \backslash K_{m_{p+1}} \quad \forall q \geq p+2 .
$$

This is impossible because the subsequence $\left\{f_{n_{q}}\right\}_{q} \rightarrow f$ in $\operatorname{Hol}\left(\Delta^{*}, X\right)$ and $K \subset K_{m_{p}} \subset$ $\operatorname{int}\left(K_{m_{p+1}}\right) \subset K_{m_{p+1}}$, so $\operatorname{dist}\left(K_{m_{p}}, U \backslash K_{m_{p+1}}\right)>0$. Hence $X_{0} \Subset U$. Thus $\left\{f_{n} ; n \geq 1\right\}$ is relatively compact in $\operatorname{Hol}\left(\Delta_{s}, U\right)$. It follows that $\left\{f_{n}\right\}$ converges uniformly on compact subsets of $\Delta_{s}$ to the mapping $g$ in $\operatorname{Hol}\left(\Delta_{s}, Y\right)$. Therefore $X$ is weakly-disc convex for $Y$. Q.E.D

Theorem 5. Let $X$ be an open complex subspace of a complex space $Y$. Suppose that $X$ is weakly hyperbolic for $Y$ and has $\Delta^{*}-E P$ for $Y$. Let $A$ be any analytic hypersurface of a 
complex manifold $M$. Let $\left\{f_{j}: M \backslash A \rightarrow X\right\}_{j=1}^{\infty}$ be a sequence of holomorphic mappings which converges uniformly on compact subsets of $M \backslash A$ to a holomorphic mapping $f: M \backslash A \rightarrow X$.

Then there are unique holomorphic extensions $\bar{f}_{j}: M \rightarrow Y$ and $\bar{f}: M \rightarrow Y$ of $f_{j}$ and $f$ respectively over $M$. Moreover, $\left\{\bar{f}_{j}\right\}_{j=1}^{\infty}$ converges uniformly on compact subsets of $M$ to $\bar{f}$.

Proof. Take any $f \in \operatorname{Hol}(M \backslash A, X)$. We may assume that $A$ is nonsingular; i.e., we extend $f$ to $M \backslash S(A)$ then to $M \backslash S(S(A))$ and so on. Here $S(Z)$ denotes the singular locus of $Z$.

By localizing the mapping $f$, we may assume that $M=\Delta^{m}=\Delta^{m-1} \times \Delta$ and $A=$ $\Delta^{m-1} \times\{0\}$.

For each $z^{\prime} \in \Delta^{m-1}$, consider the holomorphic mapping $f_{z^{\prime}}: \Delta^{*} \rightarrow X$ given by $f_{z^{\prime}}(z)=$ $f\left(z^{\prime}, z\right)$ for all $z \in \Delta^{*}$. Since $X$ has the $\Delta^{*}$-EP for $Y$, there exists a holomorphic extension $\bar{f}_{z^{\prime}}$ : $\Delta \rightarrow Y$ of $f_{z^{\prime}}$ for each $z^{\prime} \in \Delta^{m-1}$. Define the mapping $\bar{f}: \Delta^{m-1} \times \Delta \rightarrow Y$ by $\bar{f}\left(z^{\prime}, z\right)=\bar{f}_{z^{\prime}}(z)$ for all $\left(z^{\prime}, z\right) \in \Delta^{m-1} \times \Delta$. It suffices to prove that $\bar{f}$ is continuous at $\left(z_{0}^{\prime}, 0\right) \in \Delta^{m-1} \times \Delta$.

Indeed, assume that $\left\{\left(z_{k}^{\prime}, z_{k}\right)\right\} \in \Delta^{m-1} \times \Delta$ such that $\left\{\left(z_{k}^{\prime}, z_{k}\right)\right\} \rightarrow\left(z_{0}^{\prime}, 0\right)$. Put $\sigma_{k}=\bar{f}_{z_{k}^{\prime}}$ for each $k \geq 1$ and $\sigma_{0}=\bar{f}_{z_{0}^{\prime}}$. Then the sequence $\left\{\left.\sigma_{k}\right|_{\Delta^{*}}\right\}$ converges uniformly on compact subsets to the mapping $\left\{\left.\sigma_{0}\right|_{\Delta^{*}}\right\}$ in $\operatorname{Hol}\left(\Delta^{*}, X\right)$. Since $X$ is weakly hyperbolic for $Y$ and has the $\Delta^{*}$-EP for $Y$, by Proposition $6, X$ is weakly disc-convex for $Y$. It implies that the sequence $\left\{\sigma_{k}\right\}$ converges uniformly to the mapping $\sigma_{0}$ in $\operatorname{Hol}(\Delta, Y)$. Therefore, $\left\{\sigma_{k}\left(z_{k}\right)=\bar{f}\left(z_{k}^{\prime}, z_{k}\right)\right\} \rightarrow \sigma_{0}(0)=\bar{f}\left(z_{0}^{\prime}, 0\right)$. Hence, $\bar{f}$ is continuous at $\left(z_{0}^{\prime}, 0\right)$. It implies that every holomorphic mapping $f: M \backslash A \rightarrow X$ extends holomorphically over $M$.

Let $\left\{f_{k}\right\} \in \operatorname{Hol}(M \backslash A, X)$ be such that $\left\{f_{k}\right\} \rightarrow f_{0}$ in $\operatorname{Hol}(M \backslash A, X)$. We will show that $\left\{\bar{f}_{k}\right\} \rightarrow \bar{f}_{0}$ in $\operatorname{Hol}(M, Y)$.

Let $z_{0}$ be an arbitrary point of $A$. By localizing our assertion, we may assume that $M=\Delta^{m}=\Delta^{m-1} \times \Delta$ and $A=\Delta^{m-1} \times\{0\}, z_{0}=(0,0)$. Put $a_{0}=\bar{f}_{0}\left(z_{0}\right)$. We have that $\bar{f}_{0}\left(\bar{\Delta}^{m}(1 / 2)\right)=K$ is compact in $\bar{X}$. Then by the hypothesis that $X$ is weakly hyperbolic for $Y$, there exists a hyperbolic neighbourhood $U$ of $K$ in $Y$ and $a_{0} \in U$.

We first show that for an arbitrary number $0<\varepsilon<\frac{1}{2}$, there exists a neighbourhood $V_{0}$ of $z_{0}$ such that $\bar{f}_{0}\left(V_{0}\right) \subset \mathbb{B}_{U}\left(a_{0} ; \varepsilon\right):=\left\{y \in U: d_{U}\left(y, a_{0}\right)<\varepsilon\right\}$ and $\bar{f}_{k}\left(V_{0}\right) \subset \mathbb{B}_{U}\left(a_{0} ; \varepsilon\right)$ for all $k \geq k_{0}$ : Take a point $z_{1} \in \mathbb{B}_{\Delta^{m}\left(\frac{1}{2}\right)}\left(z_{0} ; \frac{\varepsilon}{3}\right) \backslash A$, where $\mathbb{B}_{\Delta^{m}\left(\frac{1}{2}\right)}\left(z_{0} ; \frac{\varepsilon}{3}\right):=\left\{z \in \Delta^{m}\left(\frac{1}{2}\right): d_{\Delta^{m}\left(\frac{1}{2}\right)}\left(z, z_{0}\right)<\frac{\varepsilon}{3}\right\}$. Then $f_{0}\left(z_{1}\right) \in \mathbb{B}_{U}\left(a_{0} ; \frac{\varepsilon}{3}\right)$. There is an integer $k_{0}=k_{0}\left(z_{1}\right)$ such that $f_{k}\left(z_{1}\right) \in \mathbb{B}_{U}\left(a_{0} ; \frac{2 \varepsilon}{3}\right)$ for all $k \geq k_{0}$. Hence we have

$$
\bar{f}_{k}\left(\mathbb{B}_{\Delta^{m}\left(\frac{1}{2}\right)}\left(z_{1} ; \frac{\varepsilon}{3}\right)\right) \subset \mathbb{B}_{U}\left(a_{0} ; \varepsilon\right) .
$$

Put $V_{0}=\mathbb{B}_{\Delta^{m}\left(\frac{1}{2}\right)}\left(z_{0} ; \frac{\varepsilon}{3}\right) \cap \mathbb{B}_{\Delta^{m}\left(\frac{1}{2}\right)}\left(z_{1} ; \frac{\varepsilon}{3}\right)$. Then $z_{0} \in V_{0}$ and $\bar{f}_{0}\left(V_{0}\right) \subset \mathbb{B}_{U}\left(a_{0} ; \varepsilon\right), \bar{f}_{k}\left(V_{0}\right) \subset \mathbb{B}_{U}\left(a_{0} ; \varepsilon\right)$ for all $k \geq k_{0}$.

Take $\varepsilon>0$ so small that $\mathbb{B}_{U}\left(a_{0} ; \varepsilon\right)$ is contained in a holomorphic local coordinate neigh- 
bourhood of $a_{0}$ in $Y$. Choose $\delta>0$ small enough such that $\bar{\Delta}_{\delta}^{m} \subset V_{0}$. Since $\left\{\left.\bar{f}_{k}\right|_{\left(\partial \Delta_{\delta}\right)^{m}}\right\}_{k=1}^{\infty}$ converges uniformly on compact subsets to $\left.\bar{f}_{0}\right|_{\left(\partial \Delta_{\delta}\right)^{m}}$, the maximum principle implies the uniform convergence of $\left\{\left.\bar{f}_{k}\right|_{\Delta_{\delta}^{m}}\right\}_{k=1}^{\infty}$ with limit $\left.\bar{f}_{0}\right|_{\Delta_{\delta}^{m}}$. Q.E.D

Theorem 5 contains the following result of D. D. Thai and P. N. Mai [13, Thm. 2], which was proved by a different method.

Corollary 1. Let $X$ be a complex subspace of a hyperbolic complex space $Y$ such that $X$ has $\Delta^{*}$ EP for $Y$. Let $A$ be an analytic hypersurface of a complex manifold $M$. Let $\left\{f_{j}: M \backslash A \rightarrow X\right\}_{j=1}^{\infty}$ be a sequence of holomorphic mappings which converges uniformly on compact subsets of $M \backslash A$ to a holomorphic mapping $f: M \backslash A \rightarrow X$.

Then there are unique holomorphic extensions $\bar{f}_{j}: M \rightarrow Y$ of $f_{j}$ and $\bar{f}: M \rightarrow Y$ of $f$ over $M$, and $\left\{\bar{f}_{j}\right\}_{j=1}^{\infty}$ converges uniformly on compact subsets of $M$ to $\bar{f}$.

By the remark b) of Definition 6 and the proof of Theorem 5 we have

Corollary 2. Let $X$ be a relatively compact and hyperbolically imbedded into a complex space $Y$. Let $M$ be a complex manifold and $A$ an analytic hypersurface of $M$. Then every holomorphic mapping $f: M \backslash A \rightarrow X$ extends to a holomorphic mapping $\bar{f}: M \rightarrow Y$.

Acknowledgments. The present work was done at the Abdus Salam International Centre for Theoretical Physics during the author's visit. The author wishes to thank the ICTP for hospitality and the stimulating research environment. The author is also grateful to Professors Do Duc Thai and Gerd Dethloff for helpful suggestions concerning this material.

\section{References}

[1] O. Alehyane and H. Amal, Propriétés d'extension et applications séparément holomorphes dans les espaces faiblement hyperboliques, (Preprint).

[2] H. Grauert and R. Remmert, Theory of Stein Spaces, Grundl. 236, Springer-Verlag, 1979.

[3] S. Kobayashi, Hyperbolic Manifolds and Holomorphic Mappings, Dekker, New York (1970).

[4] S. Kobayashi, Hyperbolic Complex Spaces, v. 318 (1998), Grundlehren der mathematischen Wissenschaften.

[5] S. Lang, Introduction to Complex Hyperbolic Spaces, Springer-Verlag, 1987.

[6] R. Narasimhan, Introduction to the Theory of Analytic Spaces, Lecture Notes in Mathematics, No. 25 (1966), Springer, Berlin.

[7] J. Noguchi and T. Ochiai, Geometric Function Theory in Several Complex Variables, Transl. Math. Monogr. vol. 80 (1990), Amer. Math. Soc. 
[8] K. Oka, Sur les fonctions analytiques de plusieurs vaiables, VII. Lemme fondamental, J. Math. Soc. Japan, 3(1951), 204-214; 259-278.

[9] B. Shabat, Introduction to Complex Analysis, Part II: Functions of Several Variables, Transl. Math. Monographs 110, Amer. Math. Soc., 1992.

[10] N. Sibony, Prolongement des fonctions holomorphes bornees et metrique de Caratheodory, Invent. Math. 29, (1975), 205-230.

[11] D. D. Thai, On the $D^{*}$-extension and the Hartogs extension, Ann. della Scuo. Nor. Super. di Pisa, Sci. Fisi. e Mate., Ser. 4 18, (1991), 13-38.

[12] D. D. Thai and N. L. Huong, On the disc-convexity of complex Banach manifolds, Ann. Pol. Math. 69, (1998), 1-11.

[13] D. D. Thai and P. N. Mai, Convergence and extension theorems in geometric function theory, Proceedings of CFCA Vol. 5, 2001, 145-162.

[14] D. D. Thai and P. J. Thomas, $D^{*}$-extension property without hyperbolicity, Indiana Univ. Math. Jour. 47, (1998), 1125-1130. 